\title{
IX. - VALEUR ALIMENTAIRE CHEZ LE POULET DANS DIVERSES CONDITIONS NUTRITIONNELLES
}

\author{
J. MOAL (1) et C. CALET ( $\left.{ }^{2}\right)$ \\ avec la collaboration technique de J. Castaing, R. Gabastou, \\ $\mathrm{Ph}$. Dascon ( $\left.{ }^{1}\right)$ et J.-M. RÉGNier ( ${ }^{3}$ ). \\ (1) Association générale des Producteurs de Mais, \\ 1. Place Samuel de Lestapis, \\ 64-Pau. \\ (2) Institut national de la Recherche agronomique, \\ Station de Recherches avicoles, $B . P . n^{0} 3$, \\ 37-Nouzilly. \\ (') Centre national d'Étude et d'Expérimentation \\ du Machinisme agricole, Parc de Tourvoie, \\ 92-Antony.

\section{RÉSUMMÉ}

Les échantillons de maïs ont été éprouvés dans plusieurs conditions nutritionnelles chez le poussin : régime de base conforme aux normes, carencé de $20 \mathrm{p}$. Ioo en lysine ou appauvri en énergie (moins 5 p. roo).

Pour l'ensemble de ces deux essais, les écarts de performances constatés (5 p. 100 au maximum) entre les divers lots de maîs proposés sont très faibles. On constate une réduction de croissance pour les maïs échauffés $(E)$ et pour la combinaison $\mathrm{F}_{80}$. Un séchage à ${ }_{4} 0^{\circ} \mathrm{C}$ ne modifie pas la valeur des maïs $E$; par contre, il semble réhabiliter les maïs $F$.

Les résultats portant sur les indices de consommation sont " contradictoires " entre les essais. Ils rendent difficiles une interprétation sûre. Ils semblent néanmoins jouer en défaveur des maĩs $\mathrm{F}$.

Il n'a pas été possible de dire si une inđisponibilité de la lysine ou de l'énergie apportées par le maïs pouvait être mise en cause.

\section{INTRODUCTION}

Divers chercheurs ont montré antérieurement que la valeur alimentaire de maïs séchés et échauffés sous des conditions expérimentales définies, pouvait être altérée de điverses façons, la principale étant l'indisponibilité partielle de certains acides aminés essentiels : 1ysine, méthionine, tryptophane (CALET et al., I960, I966 ; FMERICK et al., I96r; Foster, I962).

A l'occasion de nos travaux sur les six échantillons de maiss préparés par l'Institut technique des Céréales et des Fourrages, il nous a paru intéressant de comparer ces échantillons dans différentes conditions nutritionnelles. Il s'agissait en effet de mettre en évidence in vivo quelques-unes des particularités nutritionnelles de 
ces maïs. D'après les résultats de Godon et PetiT (V), de LARbier, Guiliaume et CALET (VII) on pouvait craindre que les divers traitements ne rendent la lysine du grain plus ou moins indisponible. De même les travaux récents de MoNTreuIL et al., I966, et de GUILBOT, LAGRANDEUR et TOLLIER, I968, laissaient prévoir une diminution de la valeur énergétique de grains mal séchés ou mal conservés.

C'est pourquoi dans un premier essai nous avons comparé l'efficacité des mêmes échantillons de mais incorporés soit dans des régimes couvrant convenablement les besoins en acides aminés du poulet (régimes " normaux 》) soit n'assurant que $80 \mathrm{p}$. Ioo des normes en lysine habituellement reconnues (régimes "carencés "). Dans un second essai, nous avons repris l'ensemble des régimes "carencés " et les avons comparés à des régimes de même composition azotée mais dont le taux énergétique était abaissé.

\section{MATÉRIEL E'T MÉTHODES}

Dans chacun des essais, I 200 coquelets a Hubbard jaune " d'un jour sont répartis dans les 24 parquets d'un poulailler au sol. Ils sont élevés pendant 56 jours. Douze lots expérimentaux répétés deux fois sont constitués. Chacun des lots reçoit un régime de type "démarrage n de o à 3 semaines et un régime "finition" de 3 à 8 semaines. Les animaux sont nourris ad libitum. Ils sont pesés individuellement à 21 et à 56 jours. A ces dates on enregistre la consommation d'aliment par parquet. Les analyses statistiques portent sur les poids, gain de poids, nourriture ingérée et indice de consommation à ces deux âges.

TABLEAU I

Expérience $n^{\circ}$ 1. - Régimes mis en cuvre pour le poulet de chair : composition et principales caractéristiques

\begin{tabular}{|c|c|c|c|c|}
\hline & \multicolumn{2}{|c|}{ Régime " normal " } & \multicolumn{2}{|c|}{$\begin{array}{l}\text { Régime " carencé " } \\
\text { en lysine }\end{array}$} \\
\hline & Démarrage & Finition & Démarrage & Finition \\
\hline Maïs $\ldots \ldots \ldots \ldots \ldots \ldots \ldots \ldots$ & 57 & 59,5 & 62,5 & 63,5 \\
\hline Farine de luzerne déshydratée $(18 \%$ & & & & \\
\hline $\mathrm{MAB})\left({ }^{1}\right) \ldots \ldots \ldots \ldots \ldots \ldots$ & 4 & 6 & 4 & 6 \\
\hline Tourteau de germes de maïs .......... & 5 & 6 & 5 & 6 \\
\hline Tourteau de soja " $44 " \ldots \ldots \ldots \ldots$ & 19 & 1 & 15,5 & - \\
\hline Tourteau de soja " 44 " enrichi en matières & & & & \\
\hline grasses $(25 \%) \ldots \ldots \ldots \ldots \ldots \ldots$ & 4 & 16 & 6 & 16 \\
\hline Farine de hareng Norvège $\approx 700 » \ldots \ldots$ & 8 & 7 & 2 & 4 \\
\hline Phosphate bicalcique $\ldots \ldots \ldots \ldots \ldots$ & - & 1 & 1 & 0,5 \\
\hline Composé minéral et vitaminique........ & 3 & 3 & 3 & 3 \\
\hline DL-méthionine à 10 p. $100 \ldots \ldots \ldots \ldots$ & 0,5 & 0,5 & 1 & 1 \\
\hline Énergie métabolisable $\mathrm{kcal} / \mathrm{kg}$ & 3040 & 3180 & 3040 & 3195 \\
\hline Protéines brutes .... & 23 & 21,1 & 20 & 18,9 \\
\hline Extrait éthéré ........ & 4,6 & 7,7 & 4,9 & 7,6 \\
\hline Cellulose $\ldots \ldots \ldots \ldots \ldots$. & 4,5 & 4,1 & 4,1 & 4,1 \\
\hline Matières minérales ...... & 5,4 & 5,5 & 5,3 & 5,2 \\
\hline Lysine $\ldots \ldots \ldots \ldots \ldots \ldots$ & 1,18 & 1,07 & 0,97 & 0,86 \\
\hline Méthionine + cystine $\ldots \ldots \ldots$ & 0,81 & 0,79 & 0,80 & 0,74 \\
\hline Tryptophane.......... & 0,25 & 0,23 & 0,23 & 0,20 \\
\hline
\end{tabular}

(1) MAB : matière azotée brute. 
- Le premier essai (régimes normaux et carencés) se déroule au début de l'été. Les poulets reçoivent les régimes dont la composition est donnée au tableau I. Outre les effets de la conservation et de la température de séchage du maïs, l'effet du taux de lysine du régime de base est étudié selon un plan factoriel ( 3 modes de conservation $\times 2$ températures $\times 2$ taux de lysine du régime).

- Le second essai a lieu à la suite du premier. Le tableau 2 donne la composition des régimes à taux énergétiques variables. De nouveau, les différents facteurs (mode de conservation et température) sont étudiés selon un plan factoriel qui tient compte du taux énergétique des régimes. On fait varier ce dernier en remplaçant $6 \mathrm{p}$. Ioo de tourteau de soja enrichi de $25 \mathrm{p}$. Ioo de matières grasses par 4,5 p. Ioo de tourteau de soja délipidé et I,5 p. Ioo de rafle de maîs.

TABLEAU 2

Expérience $n^{\circ} 2$. - Régimes utilisés : composition et principales caractéristiques

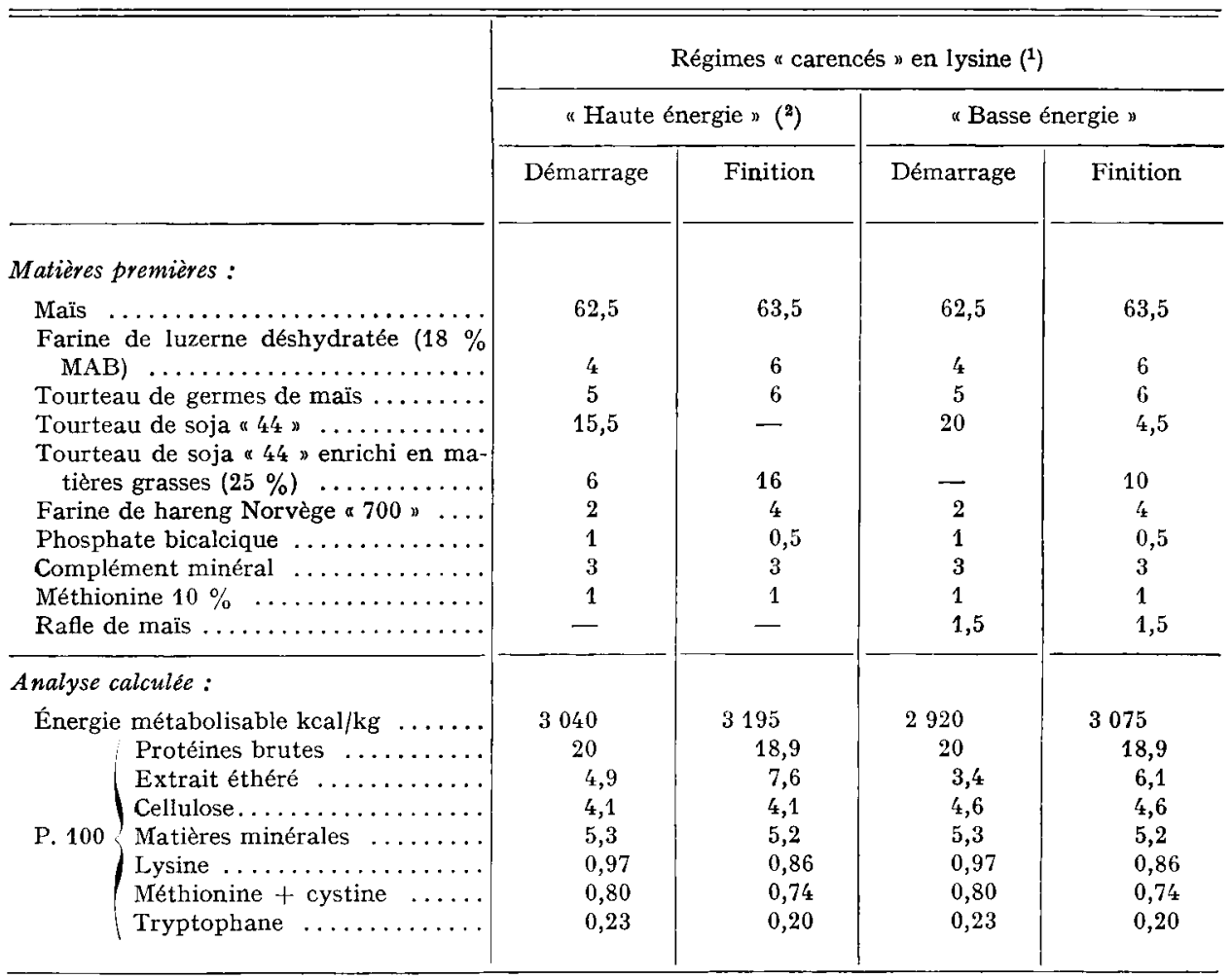

(1) C'est-à-đire à niveau en lysine inférieur de 20 p. 100 à celui d'un régime " normal ".

(2) Il s'agit en fait du régime dit "carencé " de l'essai précédent (tabl. 1).

\section{RÉSULTATS}

$$
\text { I. - Expérience } n^{\circ} \text { I }
$$

a) Poids des coquelets.

Les effets des différents traitements sont rassemblés dans le tableau 3. Ils suscitent les remarques suivantes :

- L'effet du régime carencé apparaît significatif quels que soient le traitement 
et l'âge des animaux. A huit semaines, le besoin en lysine des coquelets est encore très élevé.

\section{TABLEAU 3}

Expérience $n^{\circ} 1$. - Principaux effets des traitements du mais sur les poids à 3 semaines et à 8 semaines (g)

\begin{tabular}{|c|c|c|c|c|c|}
\hline \multirow{2}{*}{ Traitements } & \multirow{2}{*}{ Régimes } & \multicolumn{2}{|c|}{ Poids à 3 semaines } & \multicolumn{2}{|c|}{ Poids à 8 semaines } \\
\hline & & $80^{\circ} \mathrm{C}$ & $140^{\circ} \mathrm{C}$ & $80^{\circ} \mathrm{C}$ & $140^{\circ} \mathrm{C}$ \\
\hline $\mathrm{R}$ & $\begin{array}{l}\text { Normal } \\
\text { "Carencé " }\end{array}$ & $\begin{array}{l}366^{a} \\
299^{c}\end{array}$ & $\begin{array}{l}365^{a} \\
285^{a}\end{array}$ & $\begin{array}{l}1601^{a} \\
1342^{d}\end{array}$ & $\begin{array}{l}1569^{a b} \\
1328^{d}\end{array}$ \\
\hline $\mathrm{E}$ & $\begin{array}{l}\text { Normal } \\
\text { "Carencé " }\end{array}$ & $\begin{array}{l}359^{a b} \\
293^{c}\end{array}$ & $\begin{array}{l}365^{a} \\
281^{d}\end{array}$ & $\begin{array}{l}1563^{a b} \\
1323^{d}\end{array}$ & $\begin{array}{l}1567^{a b} \\
1298^{a}\end{array}$ \\
\hline $\mathrm{F}$ & $\begin{array}{l}\text { Normal } \\
\text { "Carencé " }\end{array}$ & $\begin{array}{l}351^{b} \\
293^{c}\end{array}$ & $\begin{array}{l}368^{a} \\
302^{c}\end{array}$ & $\begin{array}{l}1535^{b} \\
\text { 1. } 363^{c d}\end{array}$ & $\begin{array}{l}1577^{a b} \\
1408^{c}\end{array}$ \\
\hline \multicolumn{2}{|c|}{ Effet régime........ } & \multicolumn{2}{|c|}{$\begin{array}{c}\text { HS } \\
\text { p.p.d.s. : } 4 \mathrm{~g}\end{array}$} & \multicolumn{2}{|c|}{$\begin{array}{c}\text { HS } \\
\text { p.p.d.s. : } 18 \mathrm{~g}\end{array}$} \\
\hline \multicolumn{2}{|c|}{ Effet température $\ldots .}$. & \multicolumn{2}{|c|}{ NS } & \multicolumn{2}{|c|}{ NS } \\
\hline \multicolumn{2}{|c|}{ Effet préstockage...... } & \multicolumn{2}{|c|}{ NS } & \multicolumn{2}{|c|}{$\begin{array}{c}\text { S } \\
\text { p.p.d.s. : } 28 \mathrm{~g}\end{array}$} \\
\hline \multicolumn{2}{|c|}{$\begin{array}{l}\text { Interactions : } \\
\text { Temp. } \times \text { Régime ..... } \\
\text { Temp. } \times \text { Consommation } \\
\text { Consom. } \times \text { Régime.... }\end{array}$} & \multicolumn{2}{|c|}{$\begin{array}{l}\text { HS p.p.d.s. } 8 \mathrm{~g} \\
\text { HS p.p.d.s. } 11 \mathrm{~g} \\
\text { HS p.p.d.s. } 11 \mathrm{~g}\end{array}$} & \multicolumn{2}{|c|}{$\begin{array}{c}\text { NS } \\
\text { S p.p.d.s. } 55 \mathrm{~g} \\
\text { S p.p.d.s. } 55 \mathrm{~g}\end{array}$} \\
\hline
\end{tabular}

-- L'effet de la température est globalement non significatif aux deux âges où la mesure a été prise. Pourtant une analyse plus fine montre que l'effet " température " se manifeste seulement lorsque les animatux sont jeunes ( 3 semaines) et principalement lorsqu'ils reçoivent le régime carencé. Par rapport à $80^{\circ} \mathrm{C}$, 1a température élevée de $\mathrm{I}_{4} 0^{\circ} \mathrm{C}$ est néfaste aussi bien lorsque le maïs a été séché dès la récolte (R) qu'après un échauffement $(\mathrm{E})$. On note toutefois un effet différent pour le maïs ventilé : en régime carencé les performances du lot $F_{140}$ sont identiques à celles du lot $\mathrm{F}_{80}$ et en régime normal, elles sont supérieures. Cet effet inattendu, net à la troisième semaine, disparaît à la huitième.

Néanmoins, dans l'ensemble des lots, on note une tendance aux meilleures performances lorsque la température de séchage est basse.

- L'effet du mode de conservation est globalement non significatif sur le poids à 3 semaines alors qu'il le devient à 8 semaines. Avec le régime normal cet effet ne se manifeste que pour une température de séchage de $80^{\circ} \mathrm{C}$, et $1^{\prime}$ on assiste à une diminution du poids chez les lots recevant le maïs conservé par ventilation d'air refroidi. C'est l'inverse avec le régime carencé, où l'efficacité des maïs ventilés est la meilleure lorsque la température atteint $140^{\circ} \mathrm{C}$.

En bref, l'effet net du mode de conservation concerne le maïs ventilé avec de 1'air froid avant séchage. Quelle que soit la température de séchage, ce maïs permet 
une croissance du poulet inférieure ou au plus égale à celle des autres lots, lorsqu'il est introduit dans un régime complet et équilibré. Avec un régime carencé au contraire, ce maïs donne des résultats supérieurs à tous les autres lorsque sa température de séchage est de $140^{\circ} \mathrm{C}$.

- Ces résultats laissent prévoir les nombreuses interactions entre les facteurs étudiés. Elles sont significatives surtout à 3 semaines :

- entre la température de séchage et le taux de lysine du régime (cas des maïs F). Cette interaction se manifeste lorsque les sujets ont 3 semaines; elle disparait après.

- entre la température de séchage et le mode de conservation : elle se manifeste à tous les âges étudiés. Lorsque les maïs sont séchés à $140^{\circ} \mathrm{C}$, les performances obtenues avec le maìs échauffé tendent à s'abaisser. Par contre, cette température de séchage a un effet améliorateur sur le mais ventilé.

- entre le mode de conservation et le taux de lysine : le mais échauffé diminue les performances pondérales de façon significative lorsque le régime est "carencé "•

b) Consommations quotidiennes (tab1. 4).

On ne peut pas mettre en évidence d'effets nets des maïs sur la consommation d'aliments ; seul un faible apport de lysine dans le régime entraine une augmentation significative de la quantité moyenne d'aliment consommé pendant la période initiale (de o à 3 semaines). Cette amélioration, qui atteint 2 grammes de plus par jour

TABLEAU 4

Expérience $n^{\circ} 1$. - Quantités moyennes d'aliment consommées, g/jour/poulet

\begin{tabular}{|c|c|c|c|c|c|c|c|}
\hline \multirow{2}{*}{ Traitement } & \multirow{2}{*}{ Régime } & \multicolumn{2}{|c|}{ A 3 semaines } & \multicolumn{2}{|c|}{ De 4 à 8 semaines } & \multicolumn{2}{|c|}{ A 8 semaines } \\
\hline & & $80^{\circ} \mathrm{C}$ & $140^{\circ} \mathrm{C}$ & $80^{\circ} \mathrm{C}$ & $140^{\circ} \mathrm{C}$ & $80^{\circ} \mathrm{C}$ & $140^{\circ} \mathrm{C}$ \\
\hline $\mathbf{R}$ & $\begin{array}{l}\text { Normal } \\
\text { Carencé }\end{array}$ & $\begin{array}{l}28,4 \\
28,7\end{array}$ & $\begin{array}{l}27,9 \\
28,9\end{array}$ & $\begin{array}{l}65,6 \\
58,5\end{array}$ & $\begin{array}{l}61,7 \\
60,6\end{array}$ & $\begin{array}{l}52,3 \\
47,9\end{array}$ & $\begin{array}{l}49,6 \\
49,3\end{array}$ \\
\hline $\mathrm{E}$ & $\begin{array}{l}\text { Normal } \\
\text { Carencé }\end{array}$ & $\begin{array}{l}27,8 \\
30,2\end{array}$ & $\begin{array}{l}27,5 \\
29,4\end{array}$ & $\begin{array}{l}64,8 \\
59,1\end{array}$ & $\begin{array}{l}63,2 \\
57,8\end{array}$ & $\begin{array}{l}51,6 \\
48,8\end{array}$ & $\begin{array}{l}50,4 \\
47,7\end{array}$ \\
\hline F & $\begin{array}{l}\text { Normal } \\
\text { Carencé }\end{array}$ & $\begin{array}{l}28,1 \\
29,9\end{array}$ & $\begin{array}{l}28,3 \\
33,0\end{array}$ & $\begin{array}{l}61,1 \\
62,5\end{array}$ & $\begin{array}{l}65,7 \\
61,0\end{array}$ & $\begin{array}{l}49,3 \\
50,8\end{array}$ & $\begin{array}{l}52,3 \\
51,0\end{array}$ \\
\hline \multicolumn{2}{|c|}{$\begin{array}{l}\text { Effet significatif : } \\
\text { Régime ....... }\end{array}$} & \multicolumn{2}{|c|}{$\mathrm{s}$} & \multicolumn{2}{|c|}{ NS } & \multicolumn{2}{|c|}{ NS } \\
\hline
\end{tabular}

n'apparaît pas entre 4 et 8 semaines. Les valeurs pour la période totale ne sont pas différentes. Le traitement $\mathrm{F}$ a tendance à provoquer une augmentation légère de $1 \mathrm{a}$ consommation, surtout avec le régime "carencé ", mais l'effet n'est pas significatif.

c) Indices de consommation (tabl. 5).

L'effet principal observé concerne l'augmentation de l'indice de consommation provoquée par une diminution de la teneur en lysine. Les différences sont très importantes pendant les 3 premières semaines. Aucun autre effet n'apparaît significatif, 
TABLEAU 5

Expérience $n^{\circ} 1$. - Indices de consommation

\begin{tabular}{|c|c|c|c|c|c|c|c|}
\hline \multirow{2}{*}{ Traitement } & \multirow{2}{*}{ Régime } & \multicolumn{2}{|c|}{ A 3 semaines } & \multicolumn{2}{|c|}{ De 4 à 8 semaines } & \multicolumn{2}{|c|}{ A 8 semaines } \\
\hline & & $80^{\circ} \mathrm{C}$ & $140^{\circ} \mathrm{C}$ & $80^{\circ} \mathrm{C}$ & $140^{\circ} \mathrm{C}$ & $80^{\circ} \mathrm{C}$ & $140^{\circ} \mathrm{C}$ \\
\hline $\mathrm{R}$ & $\begin{array}{l}\text { Normal } \\
\text { Carencé }\end{array}$ & $\begin{array}{l}1,54 \\
1,91\end{array}$ & $\begin{array}{l}1,54 \\
2,03\end{array}$ & $\begin{array}{l}1,91 \\
2,02\end{array}$ & $\begin{array}{l}1,86 \\
2,09\end{array}$ & $\begin{array}{l}1,83 \\
2,00\end{array}$ & $\begin{array}{l}1,79 \\
2,07\end{array}$ \\
\hline $\mathrm{E}$ & $\begin{array}{l}\text { Normal } \\
\text { Carencé }\end{array}$ & $\begin{array}{l}1,52 \\
1,80\end{array}$ & $\begin{array}{l}1,50 \\
2,09\end{array}$ & $\begin{array}{l}1,89 \\
1,80\end{array}$ & $\begin{array}{l}1,89 \\
2,05\end{array}$ & $\begin{array}{l}1,81 \\
1,80\end{array}$ & $\begin{array}{l}1,80 \\
2,06\end{array}$ \\
\hline $\mathrm{F}$ & $\begin{array}{l}\text { Normal } \\
\text { Carencé }\end{array}$ & $\begin{array}{l}1,64 \\
2,05\end{array}$ & $\begin{array}{l}1,74 \\
2,22\end{array}$ & $\begin{array}{l}1,91 \\
2,10\end{array}$ & $\begin{array}{l}1,96 \\
2,00\end{array}$ & $\begin{array}{l}1,85 \\
2,09\end{array}$ & $\begin{array}{l}1,91 \\
2,04\end{array}$ \\
\hline $\begin{array}{r}\text { Effet significat } \\
\text { Régime .... }\end{array}$ & & & & & & & \\
\hline
\end{tabular}

et l'indice de consommation n'est pas augmenté pour les régimes contenant le maïs échauffé. Pour le maîs $F$, il semble toutefois que l'indice de consommation soit plus fort que pour les témoins, surtout pour le lot $F_{140}$, pendant les 3 premières semaines.

\section{2. - Expérience $n^{0} 2$}

a) Poids des coquelets.

Les principaux résultats sont reportés dans le tableau 6 .

TABLEAU 6

Expérience $n^{\circ} 2$. - Principaux effets des traitements du mais sur les poids à 3 semaines et à 8 semaines $(\mathrm{g})$

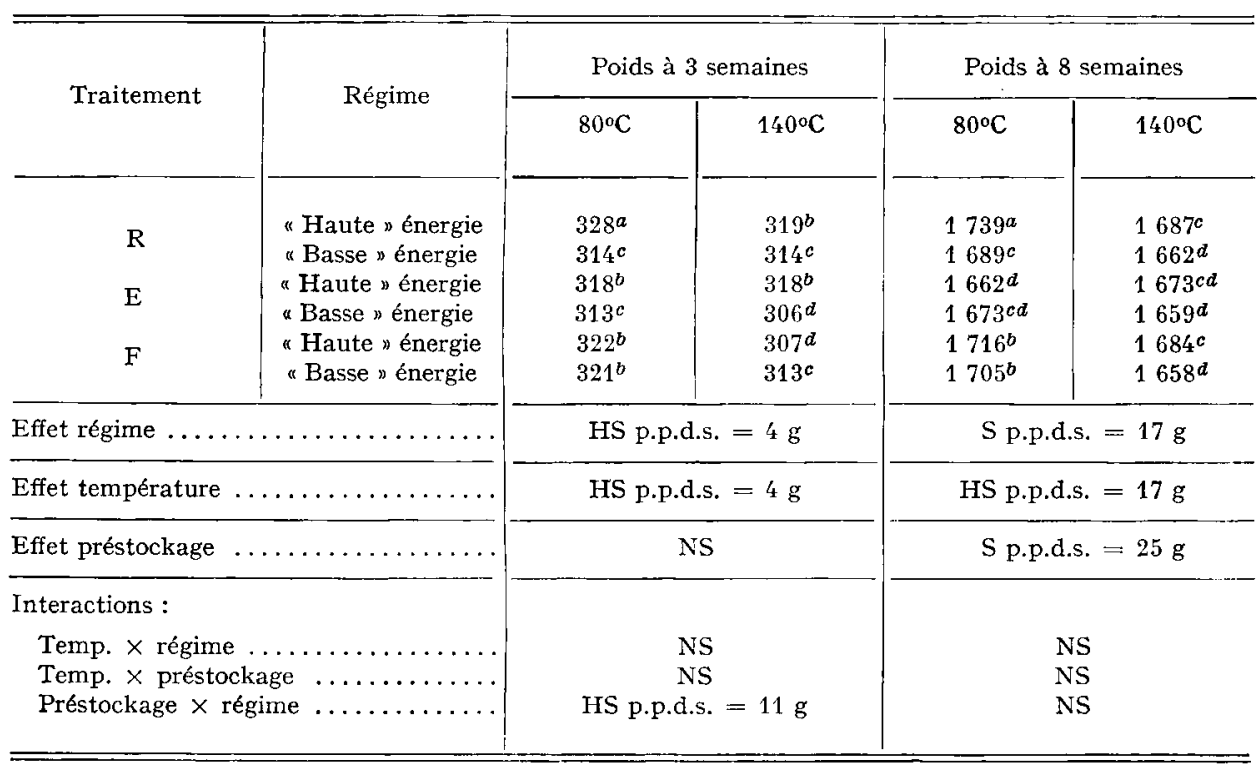


L'effet du taux énergétique y apparaît nettement. Notons toutefois que pour la moitié des lots, $1^{\prime}$ expérience $\mathrm{n}^{\circ} 2$ est une répétition de l'expérience $\mathrm{n}^{\circ} \mathrm{I}:$ il s'agit des lots recevant les régimes à faible taux énergétique. On obtient pour ces derniers les mêmes résultats que précédemment sauf pour les maïs séchés après ventilation d'air refroidi $(\mathrm{F})$ où la supériorité du lot $\mathrm{F}_{140}$ ne se manifeste plus.

Lorsque 1'on compare les échantillons de maïs introduits dans des régimes à différents taux énergétiques, le séchage à ${ } 40^{\circ} \mathrm{C}$ se montre toujours une technique défavorable. Plus encore que dans l'expérience précédente, l'échauffement spontané du maïs sans ventilation (E) est toujours préjudiciable à la croissance. Eñfin les maïs séchés à $80^{\circ} \mathrm{C}$, immédiatement après la récolte ou après ventilation, donnent des résultats équivalents et sont meilleurs que tous les autres.

\section{b) Consommations quotidiennes.}

Aucune différence significative n'apparaît entre les résultats tous très voisins de $24,6 \mathrm{~g}$ /jour pendant les 3 premières semaines, de $85,5 \mathrm{~g} /$ jour pendant les 5 dernières, de $63,8 \mathrm{~g} /$ jour pendant les 8 semaines de l'essai. Les coefficients de variation respectifs sont de $6,5,2,3$ et $2,4 \mathrm{p}$. Ioo. Ainsi les traitements du maïs n'ont eu que des effets négligeables sur ce critère, quelle que soit la nature du régime étudié.

c) Indices de consommation.

Les indices de consommation sont très voisins, pour des coefficients de variation également faibles, sauf pour la première période. Dans l'ordre indiqué pour les consommations quotidiennes, ils sont de 9,5, 2,2 et 3,0 p. Ioo. Le niveau des indices est de 2,01 .

\section{DISCUSSION}

Nous soulignerons d'entrée deux points importants pour l'utilisation pratique du maïs.

Premièrement, les maïs que l'on pourrait considérer comme "mal traités" n'ont provoqué apparemment aucun effet dépressif marqué, aucune croissance anormale, aucun comportement des animaux faisant penser à une quelconque toxicité, aucune mortalité caractérisée.

Deuxièmement, les effets des traitements du maïs n'ont pu être mis en évidence qu'en utilisant un artifice de la formulation qui permet de dévoiler les éventuels travers des échantillons. De fait, une carence frustre en lysine ou un défaut d'énergie dans le régime font apparaître quelques imperfections des maïs conservés. Toutefois, ces dernières ne sont pas importantes puisqu'elles ne se manifestent plus lorsque l'on introduit les mais dans des régimes ayant les caractéristiques des aliments distribués en pratique, c'est-à-dire lorsque les taux énergétiques des régimes de démarrage et de finition sont toujours supérieurs à $3000 \mathrm{kcal} / \mathrm{kg}$ et que les acides aminés sont entre eux dans un bon équilibre (taux de lysine toujours supérieur à I p. IOo). Cette remarque amène à penser que les normes habituellement utilisées dans l'alimentation du poulet de chair sont surestimées.

Il faut ajouter que le comportement des coquelets varie avec letur situation dans le poulailler : on observe une influence significative de la " position " ̀̀ l'intérieur 
du local expérimental sur les effets de certains traitements ; c'est pourquoi les différences entre traitements ne sont pas toujours significatives.

\section{a) Effets sur la croissance.}

Bien que significatifs, les effets des traitements subis par le maïs restent faibles, puisque les écarts maxima entre les meilleures et les plus mauvaises performances de croissance ne dépassent pas $4 \mathrm{p}$. Ioo, pour un régime normal.

Un séchage à haute température $\left(140^{\circ} \mathrm{C}\right)$ ne diminue pas le gain de poids des sujets dans le premier essai alors qu'il le fait dans le second. Cette contradiction apparente est sans doute la conséquence des autres facteurs non contrôlés des expériences. On remarque en effet que le développement des sujets est en moyenne beaucoup plus rapide dans le second essai ( $\mathrm{r} 700 \mathrm{~g}$ contre I $35^{\circ} \mathrm{g}$ ) et de ce fait les besoins nutritionnels sont plus élevés. C'est alors que l'effet de la haute température de séchage se montre toujours défavorable, vraisemblablement en rendant la lysine du maîs indisponible. Cette hypothèse est confirmée par Godon et PETIT (V) et par LARBIER et al. (VII).

Un échauffement spontané du maïs (E) a sans aucun doute un effet dépressif sur la croissance, et ceci est en parfait accord avec les résultats de CALET et TARDIF (I960).

Une conservation par ventilation refroidie $(F)$ donne des résultats plus discordants : dans l'ensemble, ils ont également un effet dépressif, paraissant tantôt plus marqué, tantôt moins marqué que celui des maiis échauffés. Dans certains cas même, ils sont égaux, sinon supérieurs aux témoins. En fait :

- séchés à $80^{\circ} \mathrm{C}$, tout joue en leur défaveur, dans le premier essai du moins;

- séchés à $14^{\circ} \mathrm{C}$, ils donnent de bons résultats, mais avant tout lorsqu'ils ont été distribué en régime carencé.

b) Effets sur la consommation.

Il est hors de doute qu'un apport insuffisant de lysine conduit les poulets à augmenter leur consommation. Nous retrouvons là les résultats de CALET et de LAMBILI, (Ig60).

Mais l'influence des divers traitements subis par le mais est beaucoup plus faible ; elle ne s'exprime que par des tendances et seulement dans la première expérience, le traitement $F_{140}$ se révélant le plus mauvais.

\section{CONCLUSION}

L'effet des divers traitements subis par ces mais est faible, surtout lorsqu'ils sont utilisés selon les « normes ". Il n'est pas possible d'en conclure qu'il faille négliger les méthodes de séchage et de conservation des maiss avant leur mise en vente.

Tout au plus peut-on dire dans les conditions de nos expériences :

- que les traitements subis par le mais n'ont pas permis la formation de toxines, mais ce risque n'est pas exclu ;

- que les méthodes d'appréciation commerciales du mais, qui concluent, exception faite du traitement $R_{80}$, au rejet de tous les lots de maïs comme " ni sains , 
ni loyaux, ni marchands " est sans commune mesure avec les faibles dépréciations constatées de leur valeur nutritionnelle.

\section{SUMMARY}

\section{IX. - FEEDING VALUE OF MAIZE FOR THE CHICKEN UNDER DIFFERENT NUTRITIONAL CONDITIONS}

The maize samples were tested in the chicken under various nutritional conditions i.e. a basal diet that was either deficient in lysine (20 p. I00 below the mean requirement), or very poor in energy.

As regards these two trials, there were only small differences (maximum 5 p. Ioo) between the performances obtained with the various maize samples. A decrease in growth was noticed when using heated maize $(E)$ and the $F_{80}$ combination. Drying at $1_{4} 0^{\circ} \mathrm{C}$ does not modify the value of the $\mathrm{E}$ maize, but it seems to improve that of the $\mathrm{F}$ maize. There was a contradiction between the trials regarding the food conversion ratios, and therefore it is difficult to give an accurate interpretation of the results obtained; it seems, however, to be against the $F$ maize samples.

It was not possible to determine whether the cause of this was either that the lysine or the energy supplied with the maize was not available.

\section{RÉFÉRENCES BIBLIOGRAPHIQUES}

Calet C., de Lambilly H., r96o. Étude de la valeur alimentaire du maïs séché artificiellement pour la croissance du poussin. I. Influence du mode de séchage sur la disponibilité des acides aminés. Ann. Zootech., 9, $181-184$.

Calet C., Tardif H., I96o. Étude de la valeur alimentaire du maïs séché artificiellement pour la croissance du poussin. II. Influence de la durée qui sépare la récolte du séchage. Ann. Zootech., 9, 349354.

Calet C., Mercier C, I966. Influence des conditions de production, de récolte et de stockage sur la composition biochimique des céréales. VI. Valeur nutritionnelle globale du maïs. Ann. Nutr. Alim., $20,24 I-256$.

Emerick R. J., Carlson C. W., Winterfeld H. L., ig6r, Effect of Heat Drying upon the Nutritive Value of Corn. Poult. Sci., 40, 99 I-994.

Foster G. H., I962. Report Effects of Drying on Corn Quality. Feedstuffs, 34, 22.

Lagrandeur G., Toldier M. Th., Guilbot A., I968. Évolution de certains constituants du grain de mais en fonction de diverses conditions de stockage et de séchage. Inds. Alim. Agric., Paris, 85, 837-842.

Montreuil J., Monsigny M., Leclerq-Ducastel G., Mercier Ch., Tollier M. Th., Guilbot A., I966. Influence des conditions de production, de récolte et de stockage sur la composition biochimique des céréales. II. Glucides. Ann. Nutr. Anim., 20, I65-I75. 